\title{
Computational Validation of Injection Molding Tooling by Additive Layer Manufacture to Produce EPDM Exte- rior Automotive Seals
}

\author{
I. Evans ${ }^{1}$, A. Rees ${ }^{1}$, C.A. Griffiths ${ }^{1}$ and R.L. Johnson ${ }^{1}$ \\ ${ }^{1}$ College of Engineering, Swansea University, UK
}

\begin{abstract}
During the design and development of Ethylene Propylene Diene Monomer (EPDM) exterior automotive seals prototype components can only manufactured through production tooling platforms by either injection molding or extrusion. Conse-quently, tooling is expensive and has long lead times. This paper investigates whether additive layer manufacture is a viable method for producing tooling used in injection molding of exterior automotive seals in EPDM. Specifically, a novel rapid tooling method that combines Additive Layer Manufacture (ALM) with epoxy reinforcement. Computational validation is performed whereby the mechanical properties of the tool are evaluated. The research has concluded that the novel tooling configuration would be suitable for prototyping purposes which would drastically reduce both costly and environmentally detrimental pre-manufacturing processes. This work has laid the foun-dations to implement rapid tooling technology to the injection molding of prototype EPDM parts.
\end{abstract}

Keywords: Digital Manufacturing, Additive Layer Manufacturing, Computational Modelling.

\section{Introduction}

\subsection{EPDM Tooling Platforms}

During product development it is necessary to manufacture production parts for validation and testing. Vehicle exterior seals are made from EPDM and currently, injection molding and extrusion is their only viable manufacturing method. Tooling for injection molding is traditionally machined from steel which have high costs and lead times, typically with lead times of 16-26 weeks for tooling for injection molding of EPDM exterior seals. EPDM is a very viscous material which requires high mold pressure during injection molding. Part design is often modified during product development which results in scraping or expensive modification cost of tooling.

Currently polyurethane (PU) prototype parts are producible however the resulting material properties do not fully align with EPDM which limits their application for prototyping purposes. Additive Layer Manufacture (ALM), also known as rapid prototyping (RP) or 3D printing, is capable of producing 3D geometries. However, for the application within this study the process could be used to produce tooling opposed to the final component which is the traditional utilization of 3D printing.

There are seven main categories that ALM techniques fall under; stereolithography, digital light processing, selective laser sintering, fused deposition modelling, laminated 
object manufacture, material jetting and binder jetting. Under each of these seven categories there are numerous variations, each with their own advantages and disadvantages. Material properties, part size, accuracy, surface finish and cost are some of the parameters that needs to be considered when selecting the additive layer manufacturing technique to be used [1-3].

Rapid tooling is the term used to describe the process of producing tooling using ALM, either creating the tool directly or creating a master model from which the tool is created, categorizing rapid tooling into direct and indirect [4-6]. Utilizing rapid tooling can reduce the lead time and significantly reduce cost when compared to traditional machining. During product development stage when time is at a premium rapid tooling offers a prominent alternative to traditional machining of injection molding tooling. Design changes are easier to accommodate when using rapid tooling allowing for quicker part improvements and consequentially reducing product lead time [4-6].

This paper investigates whether additive layer manufacture is a viable method for producing tooling used in injection molding of exterior automotive seals in EPDM. Specifically, a novel rapid tooling method that combines metal powder and epoxy, which uses ALM methods of selective laser sintering or binder jetting. Computational validation will be performed on ANSYS 19.1 software where the mechanical and thermal properties of the tool will be evaluated. A topological tool design will be proposed that has adequate mechanical strength and thermal properties.

\section{State-of-the-art}

Utilizing rapid tooling can greatly reduce the manufacturing cost and lead time for tool production [5, 7-11]. Tool material, accuracy, surface finish, and mold life are some limitations of rapid tooling [7, 11-13]. Accuracy, thermal conductivity, and mechanical properties of the tool have a significant influence on injection molding cycle, part quality and geometric complexity $[14,15]$.

Computer aided evaluation for rapid tooling process selection and manufacturability for injection molding has been presented by Nagahanumaiah [7]. A methodology compromising of three major steps; rapid tooling process selection, manufacturability evaluation, and mold cost estimation has been developed [7]. In addition, an integrated quality function deployment (QFD) and analytic hierarchy process (AHP) method were implemented in a visual $\mathrm{C}++$. The resulting computer aided evaluation aids in the selection of the most appropriate rapid tooling in addition to providing costing models [7].

$\mathrm{Au}$ et al., have performed $\mathrm{CAE}$ and CFD validation on rapid tooling for injection molding of plastic [16]. Also, in an investigation by Rahmati et al., metal filled stereolithography (SLA) rapid tooling cavity inserts were developed [17]. In particular, SLA is used to fabricate epoxy insert shells directly from CAD data which were then fitted into steel frames and reinforced with aluminum powder and epoxy resin mixture [17]. The research concluded that Moldflow Plastic Insight can be used to identify the optimal cooling system for the rapid tooling and COMSOS/Works can be used to evaluate the mechanical properties between solid and scaffolding assembly [17]. 
Structural analysis of a rapid prototype tooling made from photopolymer with stereolithography has been conducted by Huamin Zhou et al., [9]. The study predicted the deformation that occurs in the final part created in the rapid prototype tooling due to the thermal and mechanical loads of the filling process.

Stereolithography injection molding tooling experimental data was generated by $\mathrm{Hi}-$ masekhar et al., [15]. The data was used to evaluate the performance of the rapid prototype tool with regards to the distortion in each axis and the twist in the formed part. The validation results from the computational model were within $15 \%$ to the ex-perimental data for each measurement. In addition, the research also highlighted the importance of the tooling material thermal conductivity to ensure a quality part is cre-ated [15].

Three dimensional non-linear coupled thermo-mechanical finite element method (FEM) model has been developed by Song et al., to analyze the dimensional accuracy for casting dies using rapid tooling molds [10]. The FEM analysis is non-linear due to three main attributes; the material, geometry, and boundary conditions. In the study it was found that convergence criterion and time steps directly influence the computational accuracy of the FEM model. Within the study it was found that the simulated shrinkage ratio of the part casted in the rapid tooling mold was $1.108 \%$ which compares closely to the experimental shrinkage ratio of $1.158 \%$ [10].

This study will investigate whether a novel rapid tooling platform produced through additive layer manufacture is a viable method for the application of exterior automotive seals in EPDM. Specifically, computational validation a rapid tool that combines metal powder and epoxy, which uses ALM methods of selective laser sintering. Computational validation will be performed where the mechanical properties of the tool will be evaluated.

\section{$3 \quad$ Methodology}

\subsection{Novel ALM rapid tooling}

In this study a novel rapid tooling concept is proposed whereby a shell produced through ALM is reinforced with an epoxy. Combining both ALM and epoxy rapid tooling will deliver a unique tooling platform that must initially be validated through computational simulation to inform the design constraints. In particular, the mechanical requirements.

Figure 1 illustrates the manufacturing route for the proposed rapid tooling techniques to manufacture tooling for the application of injection molding. In particular, initially the shell will be produced via ALM to produce a near net insert (table 1). Following this a high temperature RS-2243 epoxy is then poured into the tool to act as reinforcement. The geometry used within the investigation corresponds to the current tooling format used within the production of EPDM automotive door seals. 


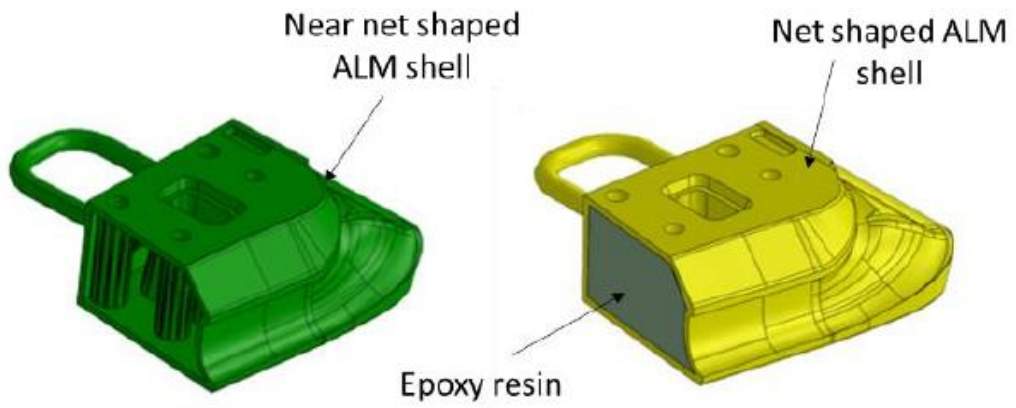

Figure 1. Process route to produce rapid tooling

The injection molding parameters used within the investigation are summarized in Table 1 .

Table 1. Typical parameters for injection moulding of EPDM exterior automotive seals.

\begin{tabular}{|c|c|}
\hline Parameter & Value \\
\hline Injection Pressure & $1400 \mathrm{bar}(140 \mathrm{MPa})$ \\
\hline Clamping Pressure & $2000 \mathrm{bar}(200 \mathrm{MPa})$ \\
\hline Temperature of Melt & $125^{\circ} \mathrm{C}$ \\
\hline Temperature of Plate & $200^{\circ} \mathrm{C}$ \\
\hline Vulcanisation Time & $85 \mathrm{~s}$ \\
\hline Melt Material & EPDM Dense 60 shore A \\
\hline
\end{tabular}

Computational Model - Mechanical simulations will be performed by ANSYS static structural system. Static structural system is chosen over the transient structural system due to the steady state nature of the application. A constant pressure of $140 \mathrm{MPa}$ will be applied at the fluid interaction face to represent the injection pressure. Fixed geometry will be applied to faces which are in contact with other parts of the tool. Figure 2 shows the physical set up of the mechanical simulation which includes a pres-sure and fixed geometries.

Contact setting between the two components of the part, the shell and reinforcement, in the computational model is set to bonded. Analysis setting will be set to run for $1 \mathrm{~s}$ with 10-time steps. Mesh sensitivity tests will be run to evaluate appropriateness of mesh characteristic to the application. 


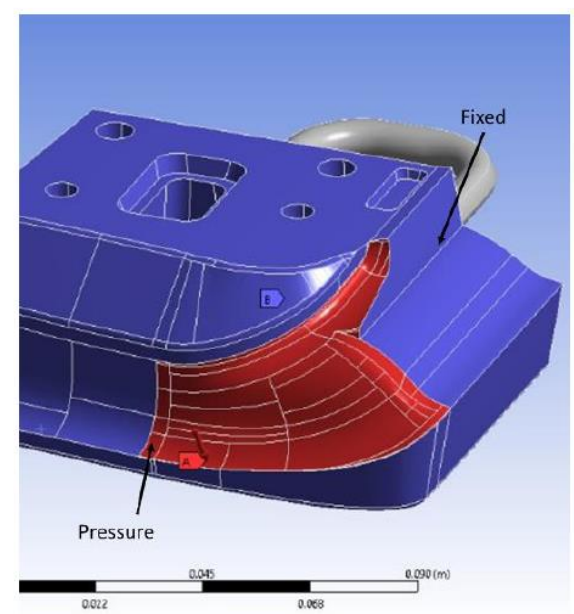

Figure 2 - Mechanical Model Setup

To investigate the effect of using different ALM materials analysis was carried out on Titanium, Aluminum and Stainless Steel. The properties are displayed in Table 2. In addition, all results from the ALM inserts were compared to a conventional P20 tool steel insert.

Table 2. Material and their properties to be used in computational simulations.

\begin{tabular}{|c|c|c|c|c|c|}
\hline Material Name & $\begin{array}{c}\text { P20 } \\
\text { Grade } \\
\text { Steel }\end{array}$ & $\begin{array}{l}\text { Ti6Al4V } \\
\text { ELI-0406 }\end{array}$ & AlSi10Mg & $\begin{array}{c}\text { SS } \\
316 \mathrm{~L}-0407\end{array}$ & $\begin{array}{c}\text { RS-2243 High } \\
\text { Temperature } \\
\text { Epoxy }\end{array}$ \\
\hline & Steel & Titanium & Aluminium & Stainless Steel & Epoxy Resin \\
\hline Use & Benchmark & Shell & Shell & Shell & Reinforcement \\
\hline Density $\left(\mathrm{g} / \mathrm{cm}^{3}\right)$ & 7.85 & 4.42 & 2.68 & 7.99 & 1.8 \\
\hline $\begin{array}{l}\text { Thermal Conductivity } \\
\left(\mathbf{W} / \mathbf{m}^{\circ} \mathbf{C}\right)\end{array}$ & 46.5 & 8 & 190 & 16.2 & 0.85 \\
\hline Melting Range $\left({ }^{\circ} \mathbf{C}\right)$ & 1460 & 1635 & 570 & 1371 & 170 \\
\hline $\begin{array}{l}\text { Coefficient of Thermal } \\
\text { Expansion }\left(\mu /{ }^{\circ} \mathbf{C}\right)\end{array}$ & 11.8 & 9 & 21 & 16.0 & 32.4 \\
\hline $\begin{array}{l}\text { Ultimate Tensile } \\
\text { Strength (MPa) }\end{array}$ & 927 & 1085 & 366 & 624 & 34.3 \\
\hline $\begin{array}{c}\text { Yield Strength } \\
(\mathrm{MPa})\end{array}$ & 716 & 985 & 220 & 494 & 27.5 \\
\hline $\begin{array}{l}\text { Youngs Modulus } \\
\text { (GPa) }\end{array}$ & 204 & 126 & 64 & 190 & 9.61 \\
\hline $\begin{array}{l}\text { Specific Heat } \\
\text { Capacity }\left(\mathrm{J} / \mathrm{kg}^{\circ} \mathrm{C}\right)\end{array}$ & 471 & 528 & 994 & 490 & 1200 \\
\hline Emissivity & 0.4 & 0.25 & 0.15 & 0.15 & 0.9 \\
\hline
\end{tabular}

For the subsequent simulations, shell thickness, shell material, and reinforcement are independent parameters, whilst displacement and stress will be the dependent parameters. Shell thicknesses will vary between $2 \mathrm{~mm}$ and $12 \mathrm{~mm}$. 


\section{$4 \quad$ Results and Discussion}

\subsection{Mesh Sensitivity Analysis}

Mesh sensitivity analysis for the mechanical simulations were performed on the benchmark tool, which is a solid part made from P20 grade steel. In particular, the mesh sensitivity analysis compared maximum and average displacement and stress, and computational time against the number of elements for a linear and quadratic type mesh is displayed in figure 3 .

The results conclude that a mesh which has 117000 elements produces converged results for both the displacement and stress simulations, irrespective of the mesh type used. Computational time for the linear mesh is less however when analyzing at the converged maximum stress results, the linear mesh results is $35 \%$ smaller than for the quadratic mesh which is a significant deviation in results. Therefore, a quadratic mesh type is found to be more accurate.
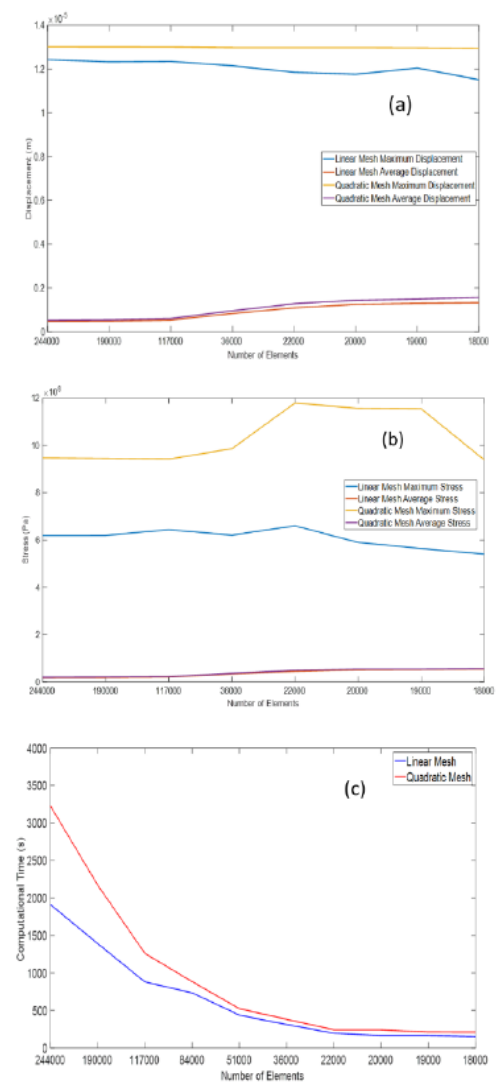

Figure 3 Mesh sensitivity analysis, comparing (a) maximum and average displacement, (b) maximum and average stress, and (c) computational time against number of elements for linear and quadratic type mesh. 


\subsection{Displacement}

Figure 4 displays the maximum displacement of inserts manufactured with different shell thicknesses, materials, and with or without reinforcement. As can be seen in figure 4 there is a large difference in maximum displacement between parts with and without reinforcement material. With the $2 \mathrm{~mm}$ thick aluminum shell the maximum displacement is 17.8 times larger in the part without reinforcement compared to the part which had reinforcement. As the shell thickness increases, there is a reduction in the difference in maximum displacement between parts with and without reinforcement. However, the part with reinforcement always has the smaller maximum displacement compared to the same part without reinforcement. This is due to the mechanical strength that the reinforcement adds the part. The results conclude that maximum displacement levels only increases marginally after a shell thickness of $6 \mathrm{~mm}$ has been reached.

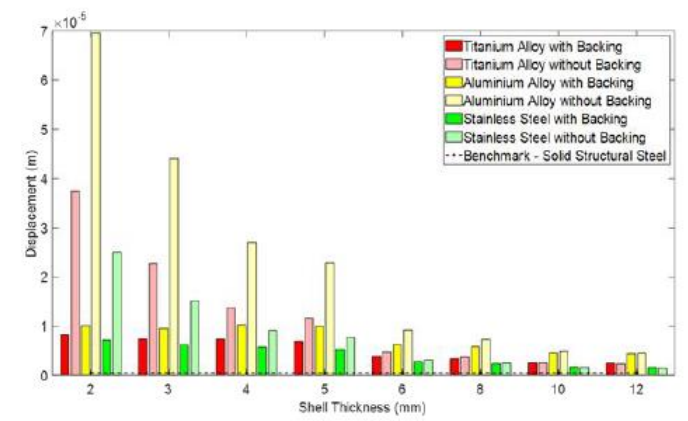

Figure 4. Maximum displacement

\subsection{Stress}

Figure 5 displays the maximum stress of parts with different thicknesses, materials, and with or without reinforcement. As seen in figure 5 the stress results follow the same trends as the displacement results in figure 4. ALM parts which have reinforcement have considerably lower maximum stress when compared to parts which have no reinforcement. It is also seen that the maximum stress in the parts decrease with an increase in shell thickness.

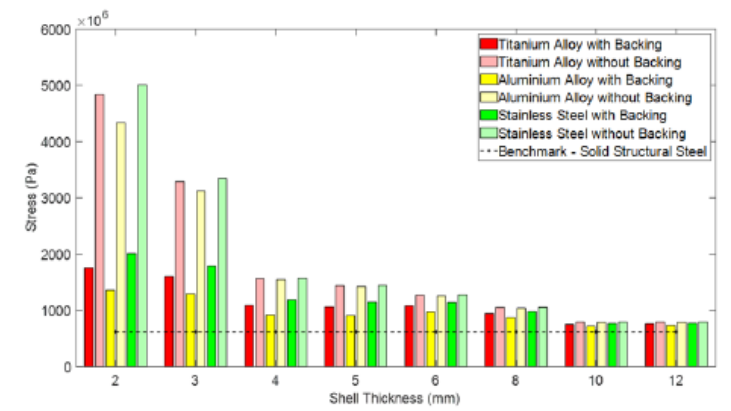

Figure 5. Maximum stress 
Comparing the ALM and benchmark parts we see very similar maximum stress in parts with shell thickness of $10 \mathrm{~mm}$ or more regardless of material. To facilitate deeper analysis the maximum stress has been compared with the material UTS, which is shown in figure 6 .

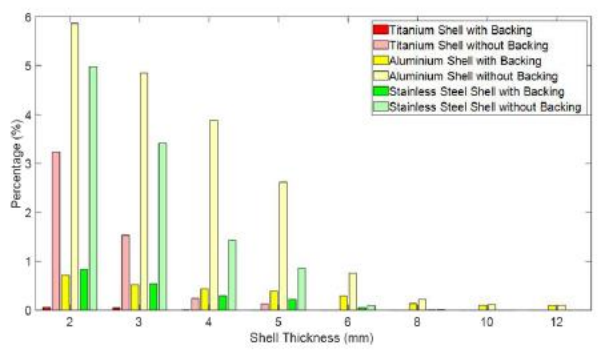

Figure 6. Maximum stress compared to UTS

As expected, the percentage of nodes with stress greater than its materials UTS decreases with an increase in shell thickness, and with the inclusion of reinforcement. Also as displayed in figure 6, the titanium shell rapid prototype tooling performs best, having significantly less nodes with stress over its material UTS compared to the aluminum and stainless-steel rapid prototype tooling. There was no exceeding of UTS stress witnessed in the titanium shell over $5 \mathrm{~mm}$ thick with reinforcement and stainless-steel shell over $10 \mathrm{~mm}$ thick with reinforcement. Furthermore, the results conclude that maximum displacement levels only increases marginally after a shell thickness of $6 \mathrm{~mm}$ has been reached.

From the results it can be concluded that ALM parts with shell thickness greater than $6 \mathrm{~mm}$ without reinforcement would also be suitable for prototype manufacture of EPDM parts using injection molding. When using a reinforced epoxy an optimum wall thickness was found to be $6 \mathrm{~mm}$.

\section{Conclusion}

A novel rapid tooling methodology has been proposed which combines metal pow-der and epoxy rapid tooling techniques. Computational validation has been performed on ANSYS 19.1 software to validate the suitability of the novel rapid tooling methodology to manufacture tooling for injection molding of EPDM exterior automotive seals. The main conclusions from the research are:

- $\quad$ Rapid prototype tooling produced by the proposed novel rapid tooling method are sufficient for prototype manufacture of EPDM parts by injection moulding.

- $\quad$ Tooling inserts produced from titanium showed the best performance. In particular, the research concludes that when using reinforcement a $6 \mathrm{~mm}$ wall is optimal.

- Mechanical properties of rapid prototype tooling $2 \mathrm{~mm}$ thick shell with reinforcement and 8mm thick shell without reinforcement are similar. Compared to the benchmark, both have similar stress results but much higher displacement results. 


\section{$6 \quad$ Future Work}

The Experimental testing is needed to validate the computational simulation findings. The characteristics of the bond between the shell and reinforcement need to be quantified. Evaluation of alternative material for the reinforcement is needed to optimize the rapid prototype tooling. Further research could evaluate the suitability of using a fluid as the reinforcement as it is incompressible.

\section{References}

1. Yan X, Gu P. A review of rapid prototyping technologies and systems. CAD Computer Aided Design. 1996

2. Pham DT, Dimov SS. Rapid manufacturing: the technologies and applications of rapid prototyping and rapid tooling. Rapid Manufacturing. Springer-Verlag London; 2001.

3. Gurr M, Mülhaupt R. Rapid Prototyping. Ref Modul Mater Sci Mater Eng. 2016

4. Rosochowski A, Matuszak A. Rapid tooling: the state of the art. J Mater Process Technol. 2000;106(1-3):191-8.

5. Chua CK, Chou SM, Wong TS. Rapid prototyping technologies and limitations. Int J Adv Manuf Technol. 1998;14:146-52

6. Kruth J-PP, Leu MCC, Nakagawa T. Progress in additive manufacturing and rapid prototyping. CIRP Ann - Manuf Technol. 1998;47(2):525-40.

7. Nagahanumaiah, Subburaj K, Ravi B. Computer aided rapid tooling process selection and manufacturability evaluation for injection mold development. Comput Ind. 2008 Mar 1;59(2-3):262-76.

8. Levy GN, Schindel R, Kruth JP. Rapid manufacturing and rapid tooling with layer manufacturing (LM) technologies, state of the art and future perspectives. CIRP Ann - Manuf Technol. 2003;52(2):589-609.

9. Zhou H, Li D. Integrated simulation of the injection molding process with stereolithography molds. Int J Adv Manuf Technol. 2006;28:53-60.

10. Song Y, Yan Y, Zhang R, Lu Q, Xu D. Three dimensional non-linear coupled thermo-mechanical FEM analysis of the dimensional accuracy for casting dies in rapid tooling. Finite Elem Anal Des. 2001 Dec 1;38(1):79-91.

11. Hague RJM. Unlocking the design potential of rapid manufacturing. Rapid manufacturing: An industrial revolution of the digital age. 2006.

12. Sood AK, Ohdar RK, Mahapatra SS. Improving dimensional accuracy of fused deposition modelling processed part using grey Taguchi method. Mater Des. 2009;30(10):4243-52.

13. Saqib S, Urbanic J. An experimental study to determine geometric and dimensional accuracy impact factors for fused deposition modelled parts: Enabling manufacturing competitiveness and economic sustainability. Berlin Heidelberg: Springer; 2012. 293-298.

14. Iyer N, Ramani K. A study of localized shrinkage in injection molding with high thermal conductivity molds. Inject Mould Technol. 2002;6(2):73-90. 
15. Himasekhar K, Lottey J, Wang KK. CAE of mold cooling in injection moulding using a three dimensional numerical simulation. $J$ Eng Ind. 1992;114(2):213-21.

16. Au KM, Yu KM. A scaffolding architecture for conformal cooling design in rapid plastic injection moulding. Int J Adv Manuf Technol. 2007;34(56):496-515.

17. Rahmati S, Dickens P. Rapid tooling analysis of Stereolithography injection mould tooling. Int J Mach Tools Manuf. 2007;47(5 SPEC. ISS.):740-7.

Tools Manuf. 2007;47(5 SPEC. ISS.):740-7. 